\title{
On the origin of the hypervelocity runaway star HD 271791
}

\author{
V. V. Gvaramadze \\ Sternberg Astronomical Institute, Moscow State University, Universitetskij Pr. 13, \\ Moscow 119992, Russia \\ email: vgvaram@mx.iki.rssi.ru
}

\begin{abstract}
We discuss the origin of the early-B-type runaway star HD 271791 and show that its extremely high velocity $\left(\simeq 530-920 \mathrm{~km} \mathrm{~s}^{-1}\right)$ cannot be explained within the framework of the binary-supernova ejection scenario. Instead, we suggest that HD 271791 attained its peculiar velocity in the course of a strong dynamical encounter between two hard, massive binaries or through an exchange encounter between a hard, massive binary and a very massive star, formed through runaway mergers of ordinary massive stars in the dense core of a young massive star cluster.
\end{abstract}

Keywords. binaries: close, stars: individual (HD 271791), stars: kinematics, galaxies: star clusters

\section{Introduction}

HD 271791 is a massive $\left(11 \pm 1 \mathrm{M}_{\odot}\right.$; Przybilla et al. 2008), extremely high-velocity runaway star with a Galactic rest-frame velocity of $\simeq 530-920 \mathrm{~km} \mathrm{~s}^{-1}$ (Heber et al. 2008), typical of the so-called hypervelocity stars (HVSs), i.e., the ordinary stars moving with peculiar velocities exceeding the escape velocity of our Galaxy (Brown et al. 2005; Edelmann et al. 2005; Hirsch et al. 2005). The existence of HVSs was predicted by Hills (1988), who showed that a close encounter between a tight binary system and the supermassive black hole $(\mathrm{BH})$ in the Galactic Centre could be responsible for ejection of one of the binary components with a velocity of up to several $1000 \mathrm{~km} \mathrm{~s}^{-1}$. Yu \& Tremaine (2003) proposed an additional mechanism for HVS production based on the interaction between a single star and a putative binary $\mathrm{BH}$ in the Galactic Centre. It is therefore plausible that some HVSs were produced in that way (Gualandris et al. 2005; Baumgardt et al. 2006; Levin 2006; Sesana et al. 2006; Ginsburg \& Loeb 2006; Lu et al. 2007; Löckmann \& Baumgardt 2008). At present, however, proper-motion measurements are not available for all but one of the known HVSs, so that it is impossible to unambiguously associate their birthplace with the Galactic Centre. HD 271791 is the only HVS with measured proper motion and all measurements show that this star was ejected from the periphery of the Galactic disc (at a galactocentric distance of $\geqslant 15 \mathrm{kpc}$; Heber et al. 2008).

There are two possible alternative explanations for the origin of HVSs. The first suggests that the HVSs attain their high peculiar velocities in the course of strong dynamical three- or four-body encounters in young and dense star clusters located in the Galactic disc (Gvaramadze 2006a, 2007; Gvaramadze et al. 2008, 2009) or in the Large Magellanic Cloud (Gualandris \& Portegies Zwart 2007). The second was proposed by Abadi et al. (2009). According to these authors, some HVSs could originate from tidal disruption of dwarf galaxies during their close passage near the Milky Way. The young age of HD 271791, $25 \pm 5$ Myr (Przybilla et al. 2008), is inconsistent with the second possibility 
since there are no indications of a recent encounter between a dwarf satellite and the Milky Way. So, we are left with the first possibility. Before discussing it in Section 4, we consider a proposal by Przybilla et al. (2008) that HD 271791 attained its peculiar velocity in the course of disintegration of a close massive binary system following a supernova (SN) explosion.

\section{HD 271791: former secondary in a massive binary system}

The spectral analysis of HD 271791 by Przybilla et al. (2008) revealed that the Fe abundance in its atmosphere is subsolar and that the $\alpha$-process elements are enhanced. The first finding is consistent with the origin of HD 271791 in the metal-poor outskirts of the Galactic disc, while the second suggests that this star was a secondary component of a massive tight binary and that its surface was polluted by nucleosynthetic products after the primary star exploded as a SN. Przybilla et al. (2008) believe that the binary-SN explosion could be responsible not only for the $\alpha$ enhancement in HD 271791 but also for the extremely high space velocity of the star. Below, we outline their scenario.

The large separation of HD 271791 from the Galactic plane $(\simeq 10 \mathrm{kpc})$, along with the proper-motion measurements (Heber et al. 2008), implies that the time of flight of this B2III star is comparable to its evolutionary age, which in turn implies that the star was ejected within several Myr after its birth in the Galactic disc. The ejection event was connected with disruption of a massive tight binary following the SN explosion. The original binary was composed of a primary star of mass of $\geqslant 55 \mathrm{M}_{\odot}$ and an early-B-type secondary (HD 271791), so that the SN explosion and binary disruption occurred early in the lifetime of HD 271791. The system was close enough to go through the commonenvelope phase before the primary exploded as a SN. During the common-envelope phase, the primary star lost most of its hydrogen envelope and the binary became a tight system composed of a Wolf-Rayet star and an early-B-type main-sequence star. At the moment of SN explosion, the mass of the primary star was $\leqslant 20 \mathrm{M}_{\odot}$ and the binary semimajor axis was $\sim 14 \mathrm{R}_{\odot}$ (corresponding to an orbital velocity of the secondary of $\leqslant 420 \mathrm{~km} \mathrm{~s}^{-1}$ ). The exploded star expelled $\simeq 10 \mathrm{M}_{\odot}$ of its mass while the remaining mass collapsed to $\mathrm{a} \leqslant 10 \mathrm{M}_{\odot} \mathrm{BH}$. The $\mathrm{SN}$ explosion was asymmetric enough to disrupt the system. Przybilla et al. (2008) assumed that HD 271791 was released at its orbital velocity and that, at the time of binary disruption, the vector of the orbital velocity was directed by chance along the Galactic-rotation direction. The first assumption is based on the widespread erroneous belief that runaways produced from a SN in a binary system have peculiar velocities comparable to their pre-SN orbital velocities. The second assumption is required to explain the difference between the assumed space velocity from the binary disruption and the Galactic rest-frame velocity of HD 271791 (provided that the latter is on the low end of the observed range, $530-920 \mathrm{~km} \mathrm{~s}^{-1}$ ).

In the next section, we discuss the conditions under which the secondary star could be launched into free flight at a velocity equal to its pre-SN orbital value.

\section{Binary-supernova ejection scenario}

One of two basic mechanisms producing runaway stars is based on a $\mathrm{SN}$ explosion in a massive, tight binary system (Blaauw 1961). After the primary star exploded as a SN, the binary system could disintegrate if the system lost more than half of its pre-SN mass (Boersma 1961) and/or the SN explosion was asymmetric (so that the stellar remnant, either a neutron star, NS, or a BH, received at birth a kick velocity exceeding the escape velocity from the system; Stone 1982; Tauris \& Takens 1998). 
In the case of binary disruption following a symmetric SN explosion, the stellar remnant is released at its orbital velocity, while the space velocity of the secondary star, $V_{\mathrm{sec}}$, is given by (Boersma 1961; Radhakrishnan \& Shukre 1985; Tauris \& Takens 1998)

$$
V_{\mathrm{sec}}=\sqrt{1-2 \frac{m_{1}+m_{2}}{m_{1}^{2}}} V_{\mathrm{orb}}
$$

where $m_{1}>2+m_{2}, m_{1}=M_{1} / M_{\mathrm{co}}, m_{2}=M_{2} / M_{\mathrm{co}}, M_{1}$ and $M_{2}$ are the pre-SN masses of the primary and the secondary stars, $M_{\text {co }}$ is the mass of the compact object formed in the SN explosion, $V_{\text {orb }}=\left[G M_{1}^{2} /\left(M_{1}+M_{2}\right) a\right]^{1 / 2}$ is the orbital velocity of the secondary star, $G$ is the gravitational constant and $a$ is the binary semimajor axis. It follows from Equation (2.1) that $V_{\mathrm{sec}} \simeq V_{\mathrm{orb}} \simeq\left(G M_{1} / a\right)^{1 / 2}$ if $M_{1} \gg M_{2}, M_{\mathrm{co}}$.

The above consideration shows that the secondary star could achieve a high peculiar speed if one adopts a large pre-SN mass for the primary (i.e., $M_{1} \gg M_{\text {co }}$ ). However, stellar evolutionary models suggest that the pre-SN masses of stars with initial masses, from 12 to $120 \mathrm{M}_{\odot}$ do not exceed $\sim 10-17 \mathrm{M}_{\odot}$ (Vanbeveren et al. 1998 ; Meynet \& Maeder 2003). Using these figures and assuming that the pre-SN binary is as tight as possible (i.e., the secondary main-sequence star is close to filling its Roche lobe), one can estimate the maximum possible velocity achieved by a runaway star in the process of binary disruption following a symmetric SN explosion. Assuming that the SN explosion left behind a NS (i.e., $M_{\text {co }}=1.4 \mathrm{M}_{\odot}$ ) and adopting $M_{1}=10-17 \mathrm{M}_{\odot}$, one finds that a

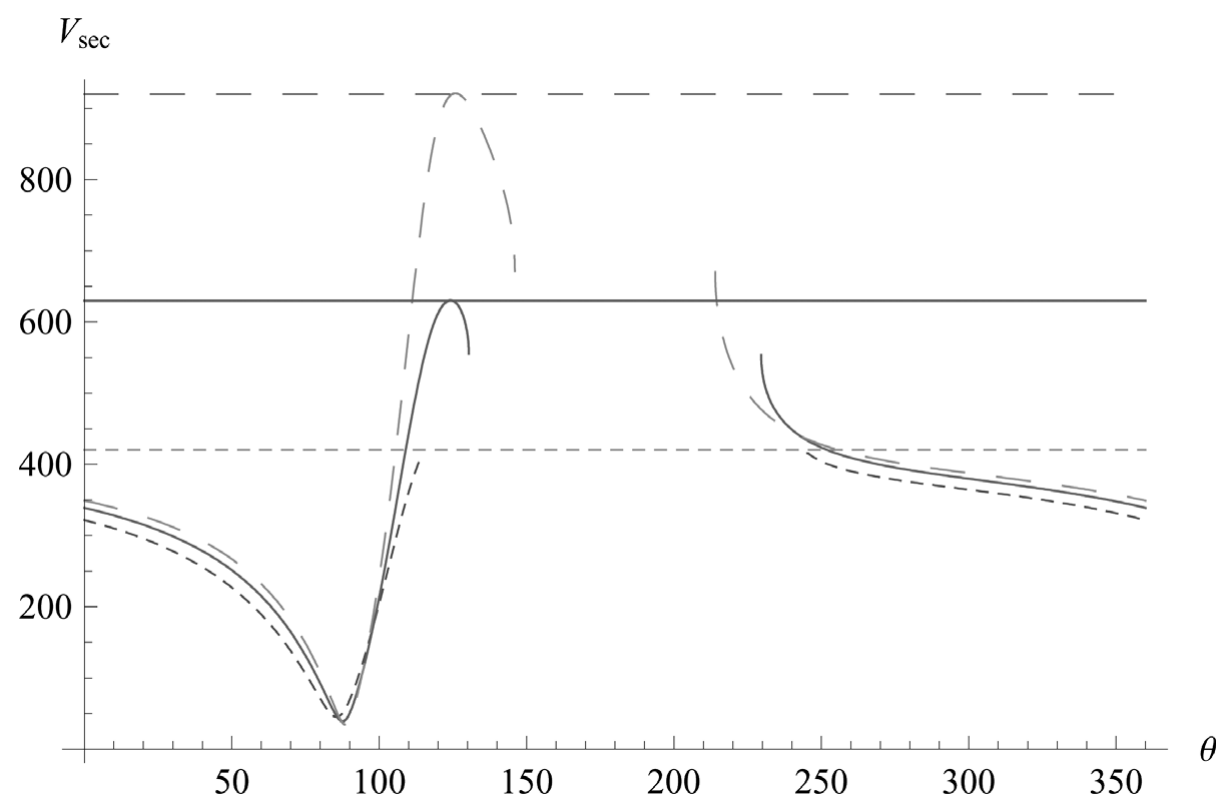

Figure 1. Peculiar velocity of the secondary star (HD 271791) as a function of the angle, $\theta$, between the kick vector and the direction of motion of the exploding star and the magnitude of the kick $\left[750 \mathrm{~km} \mathrm{~s}^{-1}\right.$ (short-dashed line), $1000 \mathrm{~km} \mathrm{~s}^{-1}$ (solid line), $1200 \mathrm{~km} \mathrm{~s}^{-1}$ (long-dashed line)]. The discontinuities in the curves correspond to a range of angles $\theta$ for which the system remains bound. The horizontal solid line indicates the Galactic rest-frame velocity of HD 271791 of $630 \mathrm{~km} \mathrm{~s}^{-1}$, corresponding to the 'best' proper motion given in Heber et al. (2008). The horizontal short- and long-dashed lines indicate, respectively, the orbital velocity of HD 271791 of $420 \mathrm{~km} \mathrm{~s}^{-1}$ [suggested by the scenario of Przybilla et al. (2008)] and the maximum possible Galactic rest-frame velocity of HD 271791 of $920 \mathrm{~km} \mathrm{~s}^{-1}$ (Heber et al. 2008). See text for details. 
$3 \mathrm{M}_{\odot}$ secondary star could attain a peculiar velocity of $\simeq 300-500 \mathrm{~km} \mathrm{~s}^{-1}$, while a $10 \mathrm{M}_{\odot}$ star could be ejected with a speed of $\simeq 350 \mathrm{~km} \mathrm{~s}^{-1}$.

Note that the pre-SN mass is maximum for stars with initial mass $\simeq 20-25 \mathrm{M}_{\odot}$ and $\geqslant 80 \mathrm{M}_{\odot}$ [see figure 6 of Meynet \& Maeder (2003)]. In the first case, the SN explosion leaves behind a NS, while in the second the stellar SN remnant is a $\mathrm{BH}$ of mass $M_{\text {co }} \geqslant$ $5 \mathrm{M}_{\odot}$ (e.g., Woosley et al. 2002). The large separation of HD 271791 from the Galactic plane implies that this massive star was ejected very soon after its birth in the Galactic disc. From this, it follows that to explain the space velocity of HD 271791 within the framework of the binary-SN scenario, one should assume that the primary was a shortlived very massive star (Przybilla et al. 2008). In this case, the stellar SN remnant is a $\mathrm{BH}$ and the $\mathrm{SN}$ ejecta are not massive enough to cause disruption of the binary system.

According to Przybilla et al. (2008), the pre-SN mass of the primary star was $\leqslant 20 \mathrm{M}_{\odot}$ (i.e., somewhat higher than the maximum mass predicted by stellar evolutionary models; see above) and the $\mathrm{SN}$ explosion left behind a $\mathrm{BH}$ of mass $\leqslant 10 \mathrm{M}_{\odot}$ (comparable to the mass of the secondary star, HD 271791), i.e., the system lost less than a half of its mass. Thus, to disrupt the binary, the SN explosion should be asymmetric. In this case, the space velocities of the BH and HD 271791 depend on the magnitude and direction of the kick imparted to the BH at birth (Tauris \& Takens 1998). To estimate $V_{\text {sec }}$, one can use equations (44)-(47) and (54)-(56) of Tauris \& Takens (1998). It follows from these equations that $V_{\mathrm{sec}}$ is maximal if the vector of the kick velocity does not strongly deviate from the orbital plane of the binary and is directed nearly towards the secondary, i.e., the angle, $\theta$, between the kick vector and the direction of motion of the exploding star is $\sim \theta_{*}=\arccos (-v / w)$, where $v=\left[G\left(M_{1}+M_{2}\right) / a\right]^{1 / 2}$ is the relative orbital velocity and $w$ is the kick velocity (see Gvaramadze 2006b).

Figure 1 shows how the direction and the magnitude of the kick affect $V_{\text {sec }}$. The three solid lines represent $V_{\text {sec }}$ calculated for the binary parameters suggested by Przybilla et al. (2008) and three kick magnitudes of $750 \mathrm{~km} \mathrm{~s}^{-1}$ (short-dashed), $1000 \mathrm{~km} \mathrm{~s}^{-1}$ (solid line) and $1200 \mathrm{~km} \mathrm{~s}^{-1}$ (long-dashed line). One can see that to launch HD 271791 at its pre-SN orbital velocity $V_{\text {orb }} \simeq 420 \mathrm{~km} \mathrm{~s}^{-1}$, the kick imparted to the BH should be at least as large as $750 \mathrm{~km} \mathrm{~s}^{-1}$. In fact, the kick magnitude should be much larger since for kicks $\simeq 750 \mathrm{~km} \mathrm{~s}^{-1}$ the kick direction must be carefully tuned (see Figure 1), i.e., $\theta$ should be either $\simeq 115^{\circ}$ or $\simeq 245^{\circ}$ (note that for $115^{\circ} \leqslant \theta \leqslant 245^{\circ}$ the binary system remains bound). The even larger kicks of $\geqslant 1000$ and $\geqslant 1200 \mathrm{~km} \mathrm{~s}^{-1}$ are required to explain the Galactic rest-frame velocities of HD 271791 of 630 and $920 \mathrm{~km} \mathrm{~s}^{-1}$ [corresponding, respectively, to the 'best' and the maximum proper motions given in Heber et al. (2008); see also Przybilla et al. (2008)]. Although one cannot exclude the possibility that BHs attain a kick at birth, we note that there is no evidence that the kick magnitude could be as large as required by the above considerations (e.g., Nelemans et al. 1999).

Thus, we found that to explain the peculiar velocity of HD 271791 the magnitude of the kick attained by the $\leqslant 10 \mathrm{M}_{\odot}$ BH must be unrealistically large $\left(\geqslant 750 \mathrm{~km} \mathrm{~s}^{-1}\right)$, which makes the binary-SN ejection scenario highly unlikely (cf. Gvaramadze 2007; Gvaramadze \& Bomans 2008). Nevertheless, some authors still believe that "HD 271791 is a plausible runaway star produced by a supernova explosion in a massive binary system" (Bromley et al. 2009).

\section{Dynamical-ejection scenario}

The second basic mechanism responsible for the origin of runaway stars is based on dynamical three- or four-body interactions in dense stellar systems (Poveda et al. 1967; 
Aarseth 1974; Gies \& Bolton 1986). Below, we discuss two possible channels for producing high-velocity runaways within the framework of the dynamical-ejection scenario (cf. Gvaramadze 2009).

The first possibility is that the high-velocity runaways originate from the interaction between two massive, hard binaries (Mikkola 1983; Leonard \& Duncan 1990). The runaways produced in binary-binary encounters are frequently ejected at velocities comparable to the orbital velocities of the binary components (Leonard \& Duncan 1990) and occasionally they can attain a velocity as high as the escape velocity, $V_{\mathrm{esc}}=\left(2 G M_{*} / R_{*}\right)^{-0.5}$, from the surface of the most massive star in the binaries (Leonard 1991). For uppermain-sequence stars with the mass-radius relationship (Habets \& Heintze 1981), $R_{*}=$ $0.8\left(M_{*} / \mathrm{M}_{\odot}\right)^{0.7} \mathrm{R}_{\odot}$, where $R_{*}$ and $M_{*}$ are the stellar radius and mass, respectively, one has $V_{\text {esc }} \simeq 700 \mathrm{~km} \mathrm{~s}^{-1}\left(M_{*} / \mathrm{M}_{\odot}\right)^{0.15}$, so that the ejection velocity could, in principle, be as large as $\simeq 1100-1200 \mathrm{~km} \mathrm{~s}^{-1}$ if the binaries contain at least one star of mass of $20-40 \mathrm{M}_{\odot}$.

To reconcile this ejection scenario with the presence of nucleosynthetic products in the atmosphere of HD 271791, one should assume that (i) HD 271791 was a secondary component of one of the binaries involved in the encounter and (ii) by the moment of the encounter, the binary containing HD 271791 has experienced SN explosion and remained bound [i.e., the stellar SN remnant $(\mathrm{BH})$ received a small or no kick at birth]. The requirement that HD 271791 was a member of a post-SN binary should also be fulfilled in the second dynamical process discussed below.

The second possibility is that the high-velocity runaway stars attain their peculiar velocities in the course of close encounters between massive, hard binaries and a very massive star (Gvaramadze 2007; Gvaramadze et al. 2009), formed through runaway collisions of ordinary massive stars in dense star clusters (e.g., Portegies Zwart et al. 1999). A close encounter with the very massive star results in tidal breakup of the binary, after which one of the binary components becomes bound to the very massive star while the second recoils with a high velocity, given by (Hills 1988)

$$
V_{\infty} \simeq 500 \mathrm{~km} \mathrm{~s}^{-1}\left(M_{\mathrm{VMS}} / 100 \mathrm{M}_{\odot}\right)^{1 / 6}\left(a^{\prime} / 30 \mathrm{R}_{\odot}\right)^{-1 / 2}\left(M_{1} / 10 \mathrm{M}_{\odot}\right)^{1 / 3},
$$

where $M_{\mathrm{VMS}}$ is the mass of the very massive star and $a^{\prime}$ is the post-SN binary semimajor axis. It follows from Equation (4.1) that, to explain the peculiar velocity of HD 271791 of $\simeq 400-600 \mathrm{~km} \mathrm{~s}^{-1}$, the mass of the very massive star should be $\geqslant 100-300 \mathrm{M}_{\odot}$ [the first figure corresponds to the mass of the most massive star formed in a 'normal' way in a cluster with a mass $M_{\mathrm{cl}} \simeq 10^{4} \mathrm{M}_{\odot}$ (Weidner et al. 2009)] $\dagger$. The above estimates can be supported by the results of three-body scattering experiments, which showed that $\geqslant 3$ percent of encounters between hard, massive binaries and a very massive star of mass of $200-300 \mathrm{M}_{\odot}$ produce runaways with $V_{\infty} \geqslant 500-600 \mathrm{~km} \mathrm{~s}^{-1}$ (Gvaramadze et al. 2009).

Note that the requirement that HD 271791 was a member of a post-SN binary does not contradict our proposal that this star can attain its high speed via a three-body encounter with a very massive star (i.e., with the star more massive than the primary star in the original binary). Merging of ordinary stars results in effective rejuvenation of the collision product (e.g., Meurs \& van den Heuvel 1989) so that the very massive star could still be on the main sequence when the most massive ordinary stars start to explode as SNe (e.g., Portegies Zwart et al. 1999).

$\dagger$ Simple estimates show that our Galaxy can currently host about 100 star clusters with masses $M_{\mathrm{cl}} \geqslant 10^{4} \mathrm{M}_{\odot}$ (Gvaramadze et al. 2008). 


\section{Acknowledgements}

I am grateful to L. R. Yungelson for useful discussions, to the Russian Foundation for Basic Research and the International Astronomical Union for travel grants and to the Deutsche Forschungsgemeinschaft for partial financial support.

\section{References}

Aarseth S. J. 1974, A\&\&A, 35, 237

Abadi, M. G., Navarro, J. F., \& Steinmetz, M. 2009, ApJ (Letters), 691, L63

Baumgardt, H., Gualandris, A., \& Portegies Zwart, S. 2006, MNRAS, 372, 174

Blaauw, A. 1961, Bull. Astron. Inst. Netherlands, 15, 265

Boersma, J., 1961, Bull. Astron. Inst. Netherlands, 15, 291

Bromley, B. C., Kenyon, S. J., Brown, W. R., \& Geller, M. J. 2009, ApJ, 706, 925

Brown W. R., Geller M. J., Kenyon S. J., \& Kurtz M. J. 2005, ApJ (Letters), 622, L33

Edelmann, H., Napiwotzki, R., Heber, U., Christlieb, N., \& Reimers, D. 2005, ApJ (Letters), 634, L181

Gies, D. R. \& Bolton, C. T. 1986, ApJS, 61, 419

Ginsburg, I. \& Loeb, A. 2006, MNRAS, 368, 221

Gualandris, A. \& Portegies Zwart, S. 2007, MNRAS (Letters), 376, L29

Gualandris, A., Portegies Zwart, S., \& Sipior, M. S. 2005, MNRAS, 363, 223

Gvaramadze, V. V. 2006a, in On the Present and Future of Pulsar Astronomy, IAU JD06 (IAU 26th General Assembly), \# 25

Gvaramadze, V. V. 2006b, A\&SA, 454, 239

Gvaramadze, V. V. 2007, $A \mathscr{E} A$ (Letters), 470, L9

Gvaramadze, V. V. 2009, MNRAS (Letters), 395, L85

Gvaramadze, V. V. \& Bomans, D.J. 2008, A\&SA, 485, L29

Gvaramadze, V. V., Gualandris, A., \& Portegies Zwart, S. 2008, MNRAS, 385, 929

Gvaramadze, V.V., Gualandris, A., \& Portegies Zwart, S. 2009, MNRAS, 396, 570

Habets, G. M. H. J., \& Heintze, J. R. W. 1981, A\&AS, 46, 193

Heber, U., Edelmann, H., Napiwotzki, R., Altmann, M., \& Scholz, R.-D. 2008, A\&A, 483, L21

Hills, J.G. 1988, Nature, 331, 687

Hirsch, H. A., Heber, U., O’Toole, S. J., \& Bresolin, F. 2005, A\&A (Letters), 444, L61

Leonard, P. J. T. 1991, AJ, 101, 562

Leonard, P. J. T., \& Duncan, M. J. 1990, AJ, 99, 608

Levin, Y. 2006, ApJ, 653, 1203

Löckmann, U. \& Baumgardt, H. 2008, MNRAS, 384, 323

Lu, Y., Yu, Q., Lin, D. N. C. 2007, ApJ (Letters), 666, L89

Meurs, E. J. A., van den Heuvel, E. P. J. 1989, A\&A, 226, 88

Meynet, G. \& Maeder, A. 2003, A\&A, 404, 975

Mikkola, S. 1983, MNRAS, 203, 1107

Nelemans, G., Tauris, T. M., \& van den Heuvel, E. P. J. 1999, A\&A (Letters), 352, L87

Portegies Zwart, S. F., Makino, J., McMillan, S. L. W., \& Hut, P. 1999, A\&SA, 348, 117

Poveda, A., Ruiz, J., \& Allen, C. 1967, Bol. Obs. Tonantzintla Tacubaya, 4, 86

Przybilla, N., Nieva, M. F., Heber, U., \& Butler, K. 2008, ApJ (Letters), 684, L103

Radhakrishnan, V. \& Shukre, C. S. 1985, in: G. Srinivasan \& V. Radhakrishnan (eds.), Supernovae, Their Progenitors and Remnants, p. 155, Bangalore: Indian Academy of Sciences

Sesana, A., Haardt, F., \& Madau, P. 2006, ApJ, 651, 392

Stone R. C. 1982, AJ, 87, 90

Tauris, T. M. \& Takens, R. J. 1998, A\&SA, 330, 1047

Vanbeveren, D., De Loore, C., \& Van Rensbergen, W. 1998, A $\& A$ Rev., 9, 63

Weidner, C., Kroupa, P., \& Bonnell, I. 2009, MNRAS, in press (arXiv:0909.1555)

Woosley, S. E., Heger, A., \& Weaver, T. A. 2002, Rev. Mod. Phys., 74, 1015

Yu, Q. \& Tremaine, S. 2003, ApJ, 599, 1129 